\title{
ALGORITHM FOR SIMULTANEOUS PARAMETER ESTIMATION OF A MULTI- HARMONIC SIGNAL
}

\section{Predrag B. Petrović}

University of Kragujevac, Technical Faculty Čačak, Svetog Save 65, 32000 Čačak, Serbia ( $ه$ predragp@tfc.kg.ac.rs, +38163 605 253)

\begin{abstract}
Estimating the fundamental frequency and harmonic parameters is basic for signal modelling in a power supply system. Differing from the existing parameter estimation algorithms either in power quality monitoring or in harmonic compensation, the proposed algorithm enables a simultaneous estimation of the fundamental frequency, the amplitudes and phases of harmonic waves. A pure sinusoid is obtained from an input multiharmonic input signal by finite-impulse-response (FIR) comb filters. Proposed algorithm is based on the use of partial derivatives of the processed signal and the weighted estimation procedure to estimate the fundamental frequency, the amplitude and the phase of a multi-sinusoidal signal. The proposed algorithm can be applied in signal reconstruction, spectral estimation, system identification, as well as in other important signal processing problems. The simulation results verify the effectiveness of the proposed algorithm.
\end{abstract}

Keywords: band-limited signals, fundamental frequency and Fourier coefficient estimation, signal reconstruction, finite-impulse-response (FIR) comb filter.

(C) 2012 Polish Academy of Sciences. All rights reserved

\section{Introduction}

Literature on electrical parameter measurement techniques for power system applications is both voluminous and generally accessible. The magnitude estimation of a power system signal has been an important area of research for the past few decades, and the methods have almost been standardized for the signals with known frequencies. The electrical parameter measurement of a fixed-frequency signal is a straightforward task. However, if the frequency is not known a priori, it becomes a very difficult task to accurately measure the amplitude and phase. Various numerical algorithms for power measurements are sensitive to frequency variations.

In an electric power system, an increase or decrease in frequency occurs due to disturbances in the power system. Large blocks of load are connected or disconnected, or large sources of generation go offline. Frequency variations are much more likely to occur for the loads that are supplied by a generator isolated from the utility systems (islands). Any frequency deviation from the nominal value of 50 or $60 \mathrm{~Hz}$ can substantially degrade the performance of the measurement devices that operate based on assumption of constant frequency.

Spectrum estimation of discretely sampled processes is usually based on procedure employing the fast Fourier transform (FFT). The FFT is a computationally efficient algorithm for computing discrete Fourier transformation (DFT). However, although the FFT is quite efficient under fixed-frequency conditions, it does not offer very good performance unless the sampling frequency and the fundamental frequency of the signal are synchronized. It is well known that FFT loses its accuracy under desynchronization and nonstationary conditions, 
whereas the fundamental/harmonic frequency may vary over time. These errors appear due to the orthogonal finite-impulse-response (FIR) filters having different magnitude gains at frequencies other that the nominal power frequency [1] and because the frequencies of harmonics are equal to zero of the frequency response of the FIR filter with a rectangular window, which is used in the DFT algorithms. These performance limitations are particularly troublesome when analyzing short data records, which frequently occur in practice, because many measured processes are brief. DFT may be computed efficiently in the sliding mode, by SDFT algorithms. After initialization a single DFT bin is computed with a few multiplications and additions and the complexity does not depend on the signal length, [2-3].

In a real case the coherent frequency relationships between all the frequencies contained in the input signal and the sampling frequency are not met, leading to the well-known leakage phenomena. The way used to reduce this problem is called 'windowing' and a frequencydomain method often used for estimating the multifrequency signal parameters under noncoherent sampling is the Interpolated DFT (IpDFT) method [4-5]. This method provides very accurate parameter estimates, but requires a considerable amount of computation.

To better satisfy the periodicity requirement of the FFT process, time-weighting functions, called windows and/or correction interpolation algorithms are used [6]. In this way, however, the error can only be reduced but not removed. If a window is not used, then the synchronization to the grid fundamental frequency is mandatory. Unfortunately, the phaselocked loop (PLL), as a traditional synchronization method, has a rather long response time, particularly in the presence of transient phenomena on the input signal, such as power supply frequency variations or phase jumps.

In addition to the disadvantages related to the synchronization of the sampling frequency with the frequency of the signal, the FFT has disadvantages caused by frame implementation. Thus, the FFT processes entire frames of data and cannot provide in-between data. If the calculation is done in a sliding mode, i.e. the FFT is repeatedly applied to a frame of $N$ elements computing of the last $N$-1-shifted elements of the previous frame and a single new element, then FFT requires intensive computational effort, which complicates its integration in low-cost microcontrollers

A Newton-type recursive numerical algorithm that also considers the system frequency as an unknown signal model parameter to be estimated has been proposed in [7]. It simultaneously estimates the frequency and spectra of the power system. This approach solves the problem of sensitivity to frequency variations. By the introduction of power frequency in the vector of unknown model parameters, the signal model becomes nonlinear, so strategies of nonlinear estimation are used. The recursive algorithm form is improved with a strategy of sequential tuning of the forgetting factor. By this, the proposed algorithm convergence and accuracy are significantly improved.

If the generator and the acquisition device are not synchronized, then the FIR filter with optimized frequency responses, which do not need synchronization, can be designed by the least-square (LS) technique [8]. In this case, the computational load is higher than in the synchronized case. The LS design method for large-order filters requires a considerable amount of computation that may not be completed within the available time, that is one sampling interval. Thus, these filters cannot efficiently be online adapted during frequency deviations. If we want to avoid the burden of these calculations, than a proper tabulation of the weights can be applied.

In this paper, a new technique for simultaneous estimation of local system frequency and amplitude and phase with wide frequency variation range is presented. What is needed for a realization of the proposed algorithm are only the value of sample of processed signal and its first and second derivation values. The framework consists of an oversampling analog-todigital conversion unit with a dithering process, a finite-impulse-response (FIR) comb filter 
and a higher order finite-impulse-response (FIR) digital differentiator followed by a decimator. The proposed method can be applied in the case when the fundamental frequency signal (input signal) has a range limited to the bandwidth of the first harmonic component. Differing from the existing parameter estimation algorithms, the proposed algorithm is able to estimate the signal parameters simultaneously, supposing the time-varying frequency. The simulation results verify the effectiveness of the proposed algorithm. The proposed method can be applied in precise measurements of important electric quantities such as RMS measurements of periodic signals, power and energy

Unlike the IEEE standard that was analysed in [9], the algorithm proposed in this paper is significantly more stable and free of the propagation error. Namely, when using the procedure prescribed by the standard, the amplitude errors of the fundamental will propagate through the method since the amplitudes are used to reconstruct the detected sine wave and obtain the results before they are used to determine the next harmonic parameters. Overall, the frequency and amplitude errors from the first calculation are propagated to the higher harmonics and the calculation of the $n$th harmonic will invariably be contaminated by the errors of the phases and amplitudes from previous steps.

\section{Proposed method of processing}

Let us assume that the input signal of the fundamental frequency $f$ is band-limited to the first $M$ harmonic components. This form of continuous signal with a complex harmonic content can be represented as a sum of the Fourier components as follows:

$$
x(t)=\sum_{k=1}^{M} X_{k} \sin \left(k \omega t+\varphi_{k}\right),
$$

where $\omega=2 \pi f$ represents the angular frequency in radians per second, $X_{k}$ is the amplitude value of the $k$ th harmonic, $\varphi_{k}$, is the phase angle of the $k$ th harmonic in radians, $M$ is the number of harmonic components in input signal, and $t$ is time in seconds.

To obtain a single sinusoidal signal, the frequency response of the filters must have nulls at the harmonic frequencies that are expected to be present in the signal and a unity gain at the fundamental frequency. If the frequency is not constant, then filter parameters have to be adapted online during frequency estimation. To provide satisfactory measurement, it becomes necessary to track the system frequency and apply certain corrections to the measuring algorithms and input filters. The block diagram of the adaptive algorithm that applies FIR comb filters is given in Fig. 1.

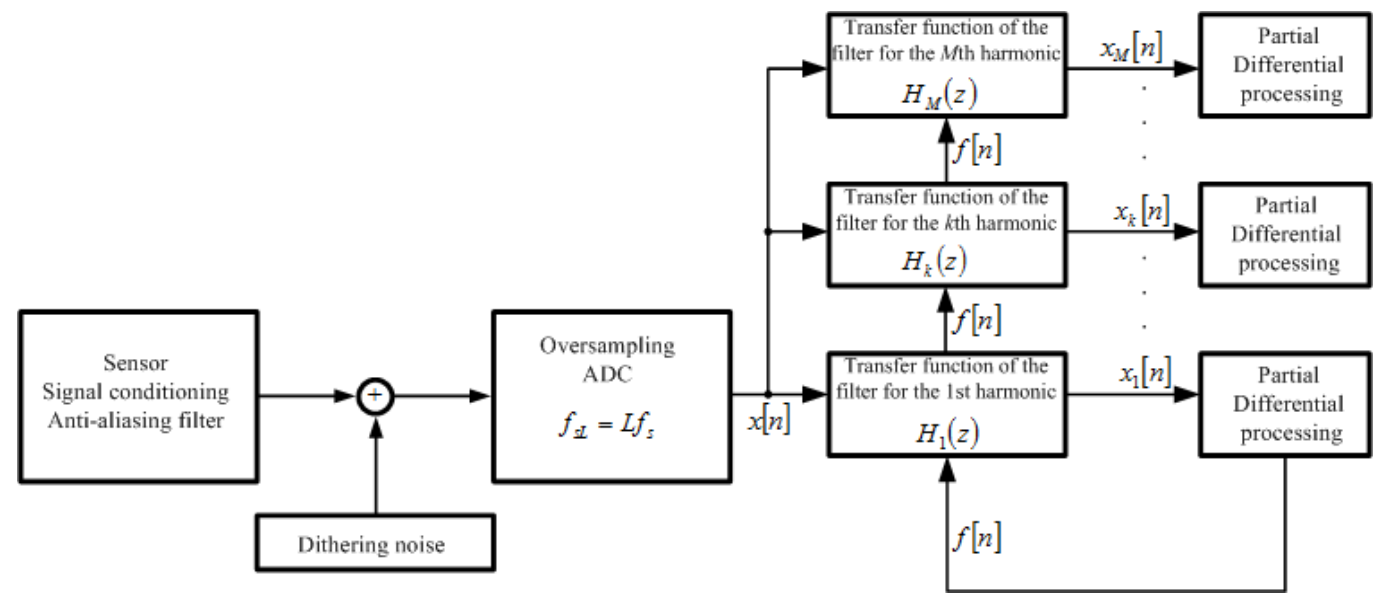

Fig. 1. Block diagram of the estimation algorithm with FIR comb filters. 
The FIR comb filters [10] consist of second-order modules that eliminate the dc component and harmonic frequencies and have unity gain at the fundamental frequency. The complete filter is realized as a cascade of all these modules. The second-order section that rejects the dc component and the frequency $f_{S} / 2\left(f_{S}\right.$ is the sampling frequency) and has a unity gain at the frequency of the $k$ th harmonics $f_{k}=k f_{1}$ is given by the following transfer function:

$$
H_{0 k}(z)=\frac{1-z^{-2}}{\left|1-z_{k}^{-2}\right|},
$$

where $\left|1-z_{k}^{-2}\right|=2 \sin \left(k \omega_{1} T\right), z^{-1}=e^{-j \omega}, \omega=2 \pi f / \omega_{S}, z_{1}^{-1}=e^{-j \omega_{1}}, \omega_{1}=2 \pi f_{1} / \omega_{S}, f_{1}$ is the fundamental frequency ( $\omega_{S}$ is the sampling frequency), and $z_{k}^{-1}=e^{-j k \omega_{1}}$.

The section that rejects the harmonics $\omega_{i}=i \omega_{1}$, and has unity gain at frequency $\omega_{k}=k \omega_{1}$ is shown as:

$$
H_{i k}(z)=\frac{1-2 \cos \left(\omega_{i} T\right) z^{-1}+z^{-2}}{\left|1-2 \cos \left(\omega_{i} T\right) z_{k}^{-1}+z_{k}^{-2}\right|},
$$

where the gain $\left|1-2 \cos \left(\omega_{i} T\right) z_{k}^{-1}+z_{k}^{-2}\right|=2 \mid \cos \left(k \omega_{1} T\right)-\cos \left(i \omega_{1} T\right), i=1,2,3, \ldots, M, i \neq k$, is used to adjust the gain for the $k$ th harmonic. $M=\left[f_{S} / 2 f_{1}\right]$ is the maximum integer part of $f_{S} / 2 f_{1}$, which is equal to the number of sections in the cascade. The transfer function of the filter for the $k$ th harmonic is given as:

$$
H_{k}(z)=H_{0 k}(z) \prod_{\substack{i=1 \\ i \neq k}}^{M} H_{i k}(z),
$$

The filter given by the transfer function (4) has a non-controlled phase shift at the fundamental frequency. In some applications, an extra phase shift of angle $\pi / 2$ has to be implemented (for example, in reactive power measurement). That can be done by an adaptive phase shifter [10]. The proposed estimation algorithm is simple because it used closed-form solutions for calculating filter coefficients. The number of sections in the cascade and data window lengths can also be changed during measurement, in dependence on the frequency changes.

It can be noticed that filters have nonunity gains at frequencies other than the nominal power system frequency. Hence, filter response adaptation during the estimation process is necessary to provide unity gain. In addition, this DFT modification with the FIR filter gives suppression of all signal harmonics which cause the leakage effect, but the FIR filter is adaptive, because its coefficients depend on the estimation of the actual frequency, and the accuracy of this algorithm depends on the accuracy of frequency estimation.

The process of estimating the frequency of multi-sinewave signals from a finite number of noisy discrete-time measurements, is an important task from both the theoretical and practical point of view. The problem has been the focus of research for quite some time and still is an active research area to date [11-15], since it is used in a wide range of applications in many fields such as control theory, relaying protection, intelligent instrumentation of power systems, signal processing, digital communications, distribution automation, biomedical engineering, radar applications, radio frequency, instrumentation and measurement, to name just a few. A list of several algorithms is reported: adaptive notch filter, time-frequency representation-based method, phase locked loop based method, eigensubspace tracking estimation, extended Kalman filter frequency estimation, internal model based method. The requirements on the frequency estimator, and so the choice of the solution, vary with the 
application, but typical issues are: accuracy, processing speed or complexity, and ability to handle multiple signals.

By differentiating the $k$ th harmonic component of the signal (1) after filtering (Fig. 1), we get:

$$
\begin{aligned}
& \frac{d\left(x_{k}(t)\right)}{d t}=\left.\frac{d\left(X_{k} \sin \left(k \omega t+\varphi_{k}\right)\right)}{d t}\right|_{t=t_{n}}=y_{1 k}\left(t_{n}\right)=y_{1 k}[n] \Rightarrow \\
& y_{1 k}\left(t_{n}\right)=k \omega X_{k} \cos \left(k \omega t_{n}+\varphi_{k}\right) \\
& \frac{d^{2}\left(x_{k}(t)\right)}{d t^{2}}=\left.\frac{d^{2}\left(X_{k} \sin \left(k \omega t+\varphi_{k}\right)\right)}{d t^{2}}\right|_{t=t_{n}}=y_{2 k}\left(t_{n}\right)=y_{2 k}[n] \Rightarrow \\
& y_{2 k}\left(t_{n}\right)=-k^{2} \omega^{2} X_{k} \sin \left(k \omega t_{n}+\varphi_{k}\right)
\end{aligned}
$$

Here, $t_{n}$ is the time moment in which the differentiation of the input analogue signal is done, and it is completely arbitrary (irregularly spaced samples). Fig. 2 shows a proposed scheme to obtain the first and second-order derivate in an oversampling system.



Fig. 2. Proposed system for signal reconstruction based on first and second-order differentiators in the oversampling system.

Based on the obtained differential value-samples, the unknown signal parameters can be calculated as:

$$
\begin{aligned}
& f_{k}\left(t_{n}\right)=k f\left(t_{n}\right)=\frac{1}{2 \pi} \sqrt{\mid \frac{y_{2 k}\left(t_{n}\right)}{x_{k}\left(t_{n}\right) \mid}} \\
& \varphi_{k}\left(t_{n}\right)=\operatorname{arctg}\left(\frac{x_{k}\left(t_{n}\right)}{y_{1 k}\left(t_{n}\right)} 2 k \pi f\left(t_{n}\right)\right)-2 k \pi f\left(t_{n}\right) t_{n} \\
& X_{k}\left(t_{n}\right)=\frac{x_{k}\left(t_{n}\right)}{\sin \left(2 k \pi f\left(t_{n}\right) t_{n}+\varphi_{k}\left(t_{n}\right)\right)}
\end{aligned}
$$

As shown in Fig. 1, a sensor picks up the signal of interest, which will be conditioned via a signal-conditioning circuit (amplifier) and band limited by an anti-aliasing filter. Then, the conditioned analog signal $x(t)$ is added with dithering noise, so that the combined signal can be fed to an ADC unit at an oversampling rate of $f_{s L}=L f_{s} \mathrm{~Hz}$ (samples/s), where $f_{s}$ and $L$ 
denote the minimum sampling rate (Nyquist sampling rate) and the oversampling factor, respectively. Each digital sample $x_{k}[n]$ is encoded using $N_{q}$ bits. The first and second-order derivates of the digitized signal are then obtained using the first and second-order FIR digital differentiators at the oversampling rate [16], which have a transfer functions designed as $H_{1 D}(z)$ and $H_{2 D}(z)$. After decimating the obtained first- and second-order derivative signals $y_{1 k}[n]$ and $y_{2 k}[n]$ by a factor $L$, we finally achieve the desired first- and second-order derivate signals $y_{1 k}[\mathrm{~m}]$ and $y_{2 k}[\mathrm{~m}]$ at the Nyquist rate of $f_{s} \mathrm{~Hz}$. Since the impact of the digital differentiator on the oversampling rate reshapes the spectrum of quantization noise, eventually resulting in its being pushed toward the high-frequency range and filtered at the same time, we can expect an improvement of signal-to-quantization-noise ratio (SQNR) for the estimated derivative signal after decimation.

The anti-aliasing filter (Fig. 1) has a bandwidth of $f_{s} / 2 \mathrm{~Hz}$. Although adding the dithering noise raises the average spectral noise floor of the original input signal, the dithering process forces the quantized error to lose its coherence with the original input signal so that the spectrum of the quantization noise becomes white and flat. Hence the oversampling technique can be applied effectively to compensate for the degraded SQNR and continue to improve the SQNR by further increasing the sampling rate. The typical amount of random wideband dithering noise, which can be provided by a noise diode or noise generator ICs usually has a root-mean-square (RMS) level equivalent to $1 / 3$ - to 1-least significant bit (LSB) voltage level. An ideal frequency response of the $k$ th-order differentiator $H_{k D}(z)$ is designated as:

$$
H_{k D}\left(e^{j \omega_{c}}\right)=\left\{\begin{array}{l}
0, \quad \text { for }-\pi \leq \omega_{c} \prec-\omega_{\max } \\
\left(j \omega_{c} / \omega_{\max }\right)^{k} \text { for }-\omega_{\max } \leq \omega_{c} \leq \omega_{\max }, \\
0, \text { for } \omega_{\max } \prec \omega_{c} \leq \pi
\end{array}\right.
$$

where $\omega_{c}=2 \pi f / f_{s L}$ is the continuous frequency of digital signal in radians, while $\omega_{\max }=2 \pi\left(f_{s} / 2\right) / f_{s L}=\pi / L$ is the maximum normalized digital frequency of the sensor signal in radians. In the oversampling system, $\omega_{\max }<<\pi$, and $H_{k D}\left(e^{j \omega_{c}}\right)$ is normalized to have a unit gain at $\omega_{\max }$. With the ideal frequency domain specification, the $k$ th-order differentiator can be designed and implemented. The effective method for designing a FIR digital differentiator using the Fourier transform design, properties of FIR differentiator coefficient, is proposed in [16], and it is out of the scope of this paper.

Due to the presence of error in determining the samples $x_{k}\left(t_{n}\right), y_{1 k}\left(t_{n}\right)$, and $y_{2 k}\left(t_{n}\right)$ in the practical applications of the proposed algorithm we need to have the best estimate of the given values, according to the criterion assumed. This can be done by means of recalculation of the values $x_{k}\left(t_{n}\right), y_{1 k}\left(t_{n}\right)$, and $y_{2 k}\left(t_{n}\right)$, through $N$ passages, ( $N$ is arbitrary). In this process we form the series $x_{k}\left(t_{n}\right)_{i}, y_{1 k}\left(t_{n}\right)_{i}$, and $y_{2 k}\left(t_{n}\right)_{i}(i=1, \ldots, N, k=1,2, \ldots, M)$, as given in the proposed algorithm. To achieve that this procedure be efficient, it is necessary to samples $x_{k}\left(t_{n}\right), y_{1 k}\left(t_{n}\right)$, and $y_{2 k}\left(t_{n}\right)$ be taken at the same points in time, through $N$ passages, compared to the detected period of the processed input signal. The random errors $\Delta_{n}$ of measurements are unbiased, $E\left(\Delta_{i}\right)=0$ have the same variance $\operatorname{var}\left(\Delta_{i}\right)=\sigma^{2}$, and are not mutually correlated. Under these assumptions, we can use the weighted average procedure for decreasing random errors in determination of observed values. The weighted average is used for measurements that are not correlated and have a varying degree of accuracy. The averages $\hat{x}_{k}\left(t_{n}\right), \hat{y}_{1 k}\left(t_{n}\right), \hat{y}_{2 k}\left(t_{n}\right)$ of the values $x_{k}\left(t_{n}\right), y_{1 k}\left(t_{n}\right)$, and $y_{2 k}\left(t_{n}\right)$ are calculated as: 


$$
\begin{gathered}
\hat{x}_{k}\left(t_{n}\right)=\frac{\sum_{i=1}^{n_{x k}} w_{x i} x_{k}\left(t_{n}\right)_{i}}{\sum_{i=1}^{n_{x}} w_{x_{k} i}} \\
\hat{y}_{j k}\left(t_{n}\right)=\frac{\sum_{n=1}^{n_{y_{j k}}} w_{y_{j i} i} y_{j k}\left(t_{n}\right)}{\sum_{i=1}^{n_{y j}} w_{y_{k j} i}} ; j=1,2 \\
\sum_{i=1}^{n_{x k}} w_{x_{k} i}=\sum_{i=1}^{n_{y j k}} w_{y_{k i} i}=\sum_{i=1}^{n_{y_{2 k}}} w_{y_{2 k} i}=N,
\end{gathered}
$$

where $w_{x_{k} i}, w_{y_{k i},}, w_{y_{2 k} i}$ are non-negative weights of series $x_{k}\left(t_{n}\right), y_{1 k}\left(t_{n}\right)$, and $y_{2 k}\left(t_{n}\right)$. The $n_{x_{k}}, n_{y_{1 k}}, n_{y_{2 k}}$ defines the numbers of different values in above series through $N$ passages. The value of $N$ will depend on the required speed of processing - the higher the $N$, the more precise the estimation of the value. After using the procedure described above to perform the estimation of the value of samples $\hat{x}_{k}\left(t_{n}\right), \hat{y}_{1 k}\left(t_{n}\right), \hat{y}_{2 k}\left(t_{n}\right)$, it is necessary to perform a recalculation of the unknown amplitudes and phases for all the harmonic components of the processed band-limited periodic signal. According to the Fourier coefficients determined in this way, it is possible to perform the calculation of the effective value of the signal, the active power and energy. When this is done, it is possible to start again the sampling of the processed signal.

\section{Simulation results}

The algorithm proposed in this paper is tested by means of the input data obtained through computer simulation. It was performed as the first and second derivation of a multi-sine signal with an 8-bit ADC resolution and an oversampling factor $L$ of 256 and $\sigma_{d}=(1 / 3) L S B$ (dithering noise is added to the analog signal before oversampling). In this way it oversampled the multi-sine signal with $4096 \cdot L$ samples.

First, an input-frequency modulated sinusoidal test signal (step frequency change from 50 to $49.8 \mathrm{~Hz}$ at $t=0.06 \mathrm{~s}$ ) was processed. White noise with $\mathrm{SNR}=60 \mathrm{~dB}$ was added to the sinusoidal signal. The test has been developed with a distorted source voltage (10\% third, $5 \%$ fifth, 3\% seventh and 2\% eleventh harmonics). The phase angles of each harmonic are randomly chosen. The results obtained confirm a good dynamic response of the algorithm for the frequency step change and accuracy. The proposed algorithm is capable of adaptively tracking time variations of the characteristics of the power signal over time. It is observed that apart from a brief transient at the time of the step change, the algorithm effectively follows the variations in frequency. As shown in Fig. 3, we have obtained a technique that provides accurate frequency estimation with an error in the range of $0.001 \mathrm{~Hz}$.

The ability of the frequency estimation over a wide range of frequency changes is investigated using sinusoidal test signals with the following time dependence $f(t)=50+0.5 \sin (10 \pi t)$ as shown in Fig. 4. Good dynamic responses can be noticed. Considering that the case simulates extreme conditions in a power system, the error can be accepted by most applications. 


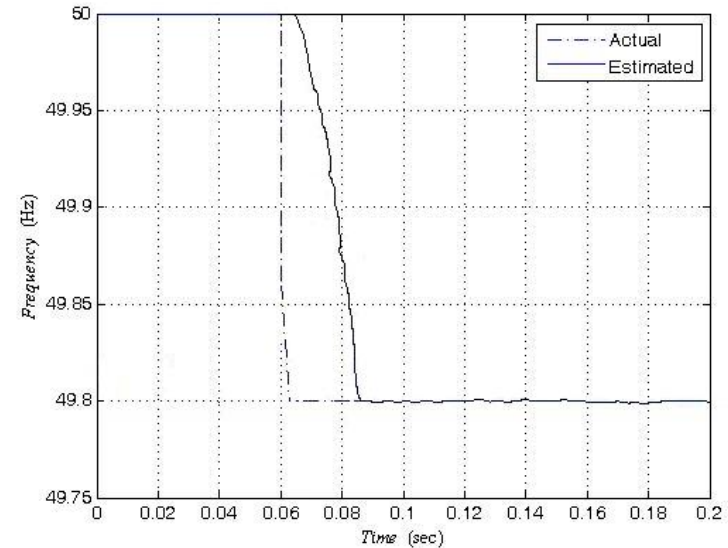

Fig. 3. Estimation for $f=50 \mathrm{~Hz}$ for $t<0.06 \mathrm{~s}$ and $f=49.8 \mathrm{~Hz}$ for $t>0.06 \mathrm{~s}$ with $\mathrm{SNR}=60 \mathrm{~dB}$ and with presence of harmonics.

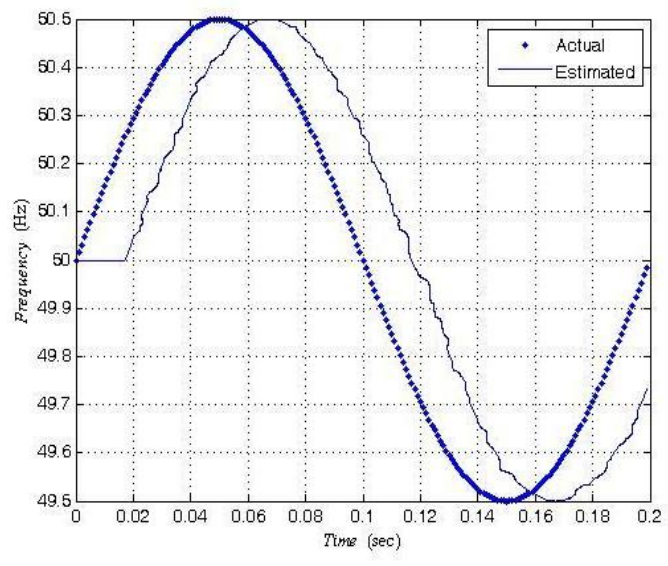

Fig. 4. Estimation for $f(t)=50+0.5 \sin (10 \pi t)$ with $\mathrm{SNR}=60 \mathrm{~dB}$ with presence of harmonics.

The effect of noise presence in the signal was studied by estimating the frequency and magnitude of signals that contain noise. A sinusoidal $50-\mathrm{Hz}$ input test signal with superimposed additive white centred Gaussian noise was used as input for the test. The random noise was selected to obtain a prescribed value of the SNR, which is defined as SNR $=20 \log (A / \sqrt{2} \sigma)$, where $A$ is the magnitude of the signal fundamental harmonics, and $\sigma$ is the noise standard deviation. Fig. 5 shows the maximum errors observed in frequency and harmonic magnitude estimates when input signals of 30,50, and $70 \mathrm{~Hz}$ having SNRs of 40, 50,60 , and $70 \mathrm{~dB}$ were used. It should be noted that, in practice, the SNR of the voltage signal obtained from a power system ranges between 50 and $70 \mathrm{~dB}$. At this level of noise, very little error is expected with the proposed technique, as depicted in Fig. 5.

a)

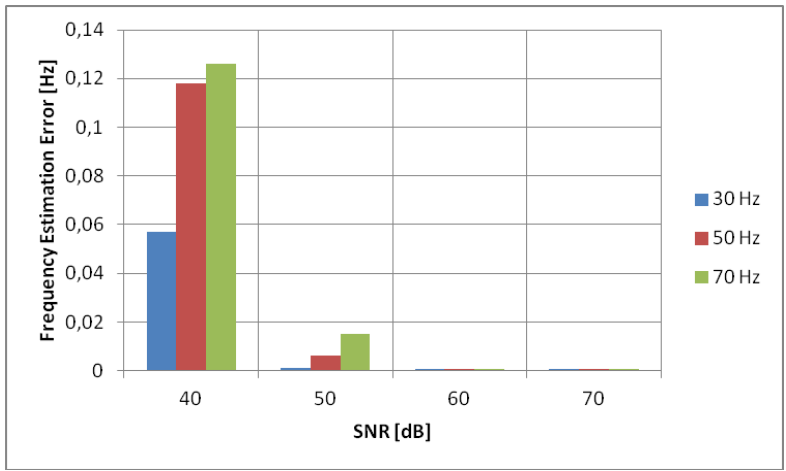

c)



b)

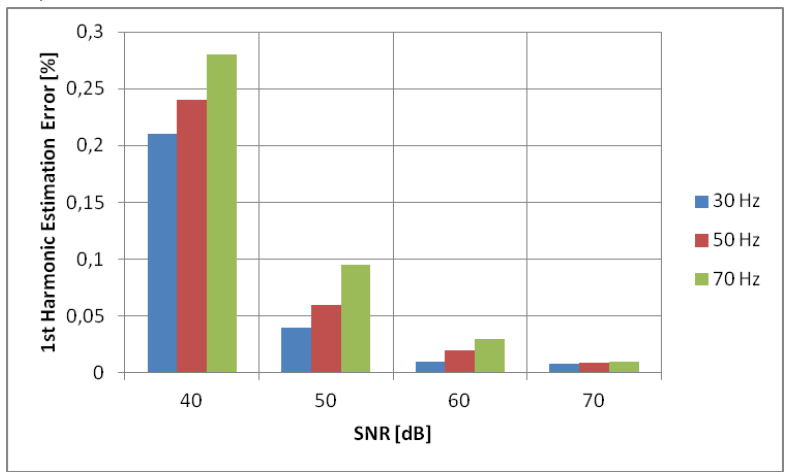

d)

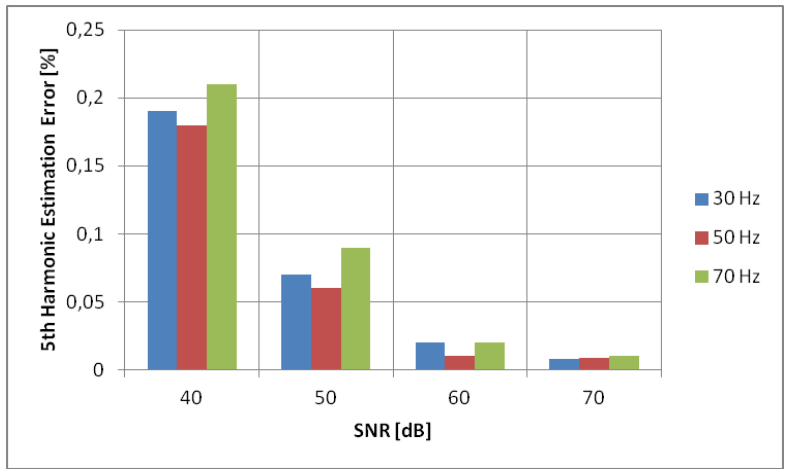

Fig. 5. Maximum estimations of errors for noisy input signals:

a) frequency; b) $1^{\text {st }}$ harmonic; c) $3^{\text {rd }}$ harmonic; d) $5^{\text {th }}$ harmonic. 
Additional testing of the proposed algorithm was carried out by simulation in the program package Matlab and SIMULINK. Standard sigma-delta ADC with an effective resolution of 24 bit was used as $\mathrm{ADC}$, and sampling rate $f_{S}=1 \mathrm{kHz}$. During the simulation, the parameters of the input signal correspond to the values given in Table 1. In the course of the simulation conducted in this way, the output PSD (Power Spectral Density) of the ideal, thermal noise affected and clock jitter affected signal was in the range of -100 to $-170 \mathrm{~dB}$ for the signal-tonoise distortion ratio (SNDR) ranging between $55 \mathrm{~dB}$ and $76 \mathrm{~dB}$.

Table 1 shows the comparison of the detection errors of the proposed estimation algorithm, FFT and continuous wavelet transformation (CWT) [17]. The presented results confirm that the fundamental frequency estimated by the proposed detection algorithm is very accurate. It can be seen that the accuracy of the proposed algorithm is within the limits that are attained in processing a signal of this form, in [18], [19], and better then the one presented in [17]. The proposed algorithm is more accurate then FFT for harmonic frequencies with decimal place.

Table 1. Comparison of simulation results by the proposed reconstruction algorithm, FFT and CWT.

\begin{tabular}{|c|c|c|c|c|c|c|c|}
\hline \multirow{2}{*}{$\begin{array}{c}\text { Harmonic } \\
{[\mathrm{Hz}]}\end{array}$} & \multirow{2}{*}{$\begin{array}{l}\text { Amplitude } \\
\text { [V] }\end{array}$} & \multicolumn{2}{|c|}{$\begin{array}{c}\text { Proposed reconstruction } \\
\text { algorithm }\end{array}$} & \multicolumn{2}{|c|}{$\begin{array}{l}\text { FFT (sampling rate }=25 \\
\mathrm{kHz} ; \\
\text { data length }=25000 ; \text { time } \\
\text { period }=1 \mathrm{~s})\end{array}$} & \multicolumn{2}{|c|}{ CWT } \\
\hline & & $\begin{array}{c}\text { Amp.error } \\
{[\%]}\end{array}$ & $\begin{array}{c}\text { Freq.error } \\
{[\%]}\end{array}$ & $\begin{array}{c}\text { Amp.error } \\
{[\%]}\end{array}$ & $\begin{array}{c}\text { Freq.error } \\
{[\%]}\end{array}$ & $\begin{array}{c}\text { Amp.error } \\
{[\%]}\end{array}$ & $\begin{array}{c}\text { Freq.error } \\
{[\%]}\end{array}$ \\
\hline 49.2 & 311 & 0.008 & 0.009 & 3.12 & 0.41 & 0.02 & 0.02 \\
\hline 102 & 280 & 0.008 & 0.006 & 0.04 & 0 & 0.03 & 0.04 \\
\hline 149.5 & 248 & 0.003 & 0.005 & 18.23 & 0.33 & 0.05 & 0.03 \\
\hline 249 & 217 & 0.005 & 0.006 & 0 & 0 & 0.15 & 0.09 \\
\hline 362 & 186 & 0.008 & 0.004 & 0 & 0 & 0.01 & 0.03 \\
\hline 442 & 155 & 0.01 & 0.01 & 0 & 0 & 0.01 & 0.03 \\
\hline 540 & 155 & 0.01 & 0.01 & 0 & 0 & 0.22 & 0.08 \\
\hline 640 & 124 & 0.02 & 0.02 & 0 & 0 & 0.21 & 0.01 \\
\hline 770 & 93 & 0.01 & 0.01 & 0.03 & 0 & 0.01 & 0.02 \\
\hline 902 & 62 & 0.02 & 0.03 & 0.02 & 0 & 0.09 & 0.06 \\
\hline
\end{tabular}

\section{Conclusion}

The estimation procedure proposed in this paper is a new algorithm for estimation of the frequency and signal parameters. The derived analytical expression opens the possibility to perform calculations with a low numeric error. The proposed algorithm is able to estimate the signal parameters simultaneously, supposing the time-varying frequency. Based on the identified parameters of the AC signals, we can establish all the relevant values in electric utilities (energy, power, RMS values). The measurement uncertainty is a function of the error in synchronization with the fundamental frequency of the processing signal (because of the non-stationary nature of the jitter-related noise and white Gaussian noise), and the error that occurs in determining the values of the samples of the processed signal. The simulation results show that the proposed algorithm can offer satisfactory precision in reconstruction of periodic signals in a real environment. 


\section{Acknowledgment}

The author wishes to thank the Ministry of Education and Science of the Republic of Serbia for its support of this work provided within the projects 42009 and OI-172057.

\section{References}

[1] Wang, F., Bollen, M. (2004). Frequency response characteristics and error estimation in RMS measurement. IEEE Trans. Power Delivery, 19(4), 1569-1578.

[2] Duda, K. (2010). Accurate, Guaranteed-Stable, Sliding DFT. IEEE Signal Processing Mag., 124-127.

[3] Jacobsen, E., Lyons, R. (2003). The sliding DFT. IEEE Signal Processing Mag., 20(2), 74-80.

[4] Wu, R.C., Chiang, C.T. (2010). Analysis of the Exponential Signal by the Interpolated DFT Algorithm. IEEE Trans. Instrum. Meas., 59(12), 3306-3317.

[5] Duda, K. (2011). Interpolation Algorithms of DFT for Parameters Estimation of Sinusoidal and Damped Sinusoidal Signals. chapter in Fourier Transform Book, In Tech. - Open Access Publisher.

[6] Wand, M., Sun, Y. (2004). A practical, precise method for frequency tracking and phasor estimation. IEEE Trans. Power Delivery, 19(4), 1547-1552.

[7] Terzija, V.V. (2003). Improved recursive Newton-type algorithms for frequency and spectra estimation in power systems. IEEE Trans. Instrum. Meas., 52(5), 1654-1659.

[8] Sidhu, T.T. (1999). Accurate measurement of power system frequency using a digital signal processing technique. IEEE Trans. Instrum. Meas., 48(1), 75-81.

[9] Arpaia, P., Cruz Serra, A., Daponte, P., Monteiro, C.L. (2001). A critical note to IEEE 1057-94 standard on hysteretic ADC dynamic testing. IEEE Trans. Instrum. Meas., 50(4), 941-948.

[10] Kuseljevic, M.D. (2008). A simple method for design of adaptive filters for sinusoidal signals. IEEE Trans. Instrum. Meas., 57(10), 2242-2249.

[11] Wu, J., Long, J., Wang, J. (2005). High-accuracy, wide-range frequency estimation methods for power system signals under nonsinusoidal conditions. IEEE Trans. Power Delivery, 20(1), 366-374.

[12] So, H.C., Chan, K.W., Chan, Y.T., Ho, K.C. (2005). Linear prediction approach for efficient frequency estimation of multiple real sinusoids: algorithms and analyses. IEEE Trans. Signal Process., 53(7), 22902305.

[13] Klein, J.D. (2006). Fast algorithms for single frequency estimation. IEEE Trans. Signal Process., 54(5), $1762-1770$

[14] El-Shafey, M.H, Mansour, M.M. (2006). Application of a new frequency estimation technique to power systems. IEEE Trans. Power Delivery, 21(3), 1045-1053.

[15] Trapero, J.R., Sira-Ramirez, H., Batlle, V.F. (2007). An algebraic frequency estimator for a biased and noisy sinusoidal signal. Signal Processing, 87(6), 1188-1201.

[16] Tan, L., Wang, L. (2011). Oversampling Technique for Obtaining Higher Order Derivative of LowFrequency Signals. IEEE Trans. Instrum. and Meas., 60(11), 3677-3684.

[17] Tse, N.C.F., Lai, L.L. (2007). Wavelet-Based Algorithm for Signal Analysis. EURASIP Journal on Advances in Signal Processing.

[18] Schoukens, J., Rolain, Y., Simon, G., Pintelon, R. (2003). Fully Automated Spectral Analysis of Periodic Signals. IEEE Trans. Instrum. Meas., 52(4), 1021-1024.

[19] Hidalgo, R.M., Fernandez, J.G., Rivera, R.R., Larrondo, H.A. (1996). A Simple Adjustable Window Algorithm to Improve FFT Measurements. IEEE Trans. Instrum. Meas., 51(1), 31-36. 